\title{
Target-present guessing as a function of target prevalence and accumulated information in visual search
}

\author{
Chad Peltier $^{1}$ - Mark W. Becker ${ }^{1}$
}

Published online: 9 February 2017

(C) The Psychonomic Society, Inc. 2017

\begin{abstract}
Target prevalence influences visual search behavior. At low target prevalence, miss rates are high and false alarms are low, while the opposite is true at high prevalence. Several models of search aim to describe search behavior, one of which has been specifically intended to model search at varying prevalence levels. The multiple decision model (Wolfe \& Van Wert, Current Biology, 20(2), 121--124, 2010) posits that all searches that end before the observer detects a target result in a target-absent response. However, researchers have found very high false alarms in highprevalence searches, suggesting that prevalence rates may be used as a source of information to make "educated guesses" after search termination. Here, we further examine the ability for prevalence level and knowledge gained during visual search to influence guessing rates. We manipulate target prevalence and the amount of information that an observer accumulates about a search display prior to making a response to test if these sources of evidence are used to inform target present guess rates. We find that observers use both information about target prevalence rates and information about the proportion of the array inspected prior to making a response allowing them to make an informed and statistically driven guess about the target's presence.
\end{abstract}

Keywords Low target prevalence - Attention - Visual search · Models of visual search

Chad Peltier

peltie11@gmail.com

1 Department of Psychology, Michigan State University, Psychology Building 316 Physics Rm 298C, East Lansing, MI 48824, USA
Target prevalence influences visual search behavior in predictable ways; as targets become rare, time spent searching and hit rate both decrease (Rich et al., 2008; Wolfe, Horowitz, \& Kenner, 2005). This is known as the low prevalence effect and is modeled by Wolfe and Van Wert (2010), multiple decision model (MDM). High miss rates in low prevalence search are attributed to a decrease in quitting threshold (Hout, Walenchok, Goldinger, \& Wolfe, 2015) and a conservative shift in decision-making criterion (Peltier \& Becker, 2016; Wolfe \& Van Wert, 2010). A low quitting threshold results in fewer item inspections before terminating search, thereby decreasing the chance of inspecting the target before termination (Rich et al., 2008; Wolfe \& Van Wert, 2010). The decision-making criterion models the decision process used to identify the currently inspected item as a target or distractor (Peltier \& Becker, 2016; Wolfe \& Van Wert, 2010). Low target prevalence results in a more conservative criterion, thereby decreasing the likelihood of a false alarm caused by misidentifying a distractor as a target (Hout et al., 2015; Peltier \& Becker, 2016). Further, the MDM posits that a target-absent response will result when the quitting threshold is reached prior to identifying a target (Wolfe \& Van Wert, 2010), suggesting all false alarms should be caused by misidentifying a distractor as the target.

Studies have found that false alarm rates are lower in low prevalence searches than high prevalence searches (Peltier \& Becker, 2016; Van Wert, Horowitz, \& Wolfe, 2009; Wolfe \& Van Wert, 2010). The MDM's proposed shift in decision criterion due to prevalence rates can explain this relationship. Under this explanation, the criterion for evaluating if a currently attended item is a target becomes more conservative as the prevalence rate decreases, decreasing the likelihood of misidentifying a distractor as a target.

However, this pattern of false alarms is also consistent with the claim that observers adjust their guessing strategies based 
on prevalence rate. Under this theory, once the quitting threshold of a trial is reached, the participant may guess that the target was present in the uninspected portion of the display. The probability of making such a guess may be impacted by the target prevalence rate. In low target prevalence searches, target-present guesses should be exceedingly rare, with the opposite being true at high prevalence.

Schwark, Macdonald, Sandry, and Dolgov (2013) provide some evidence in favor of this target-present guessing explanation. In their study, the task was to find an $X$ among many other letters. In one block, the $X$ was present on $96 \%$ of trials. In the other, the $X$ was present on $50 \%$ of trials. Observers could respond by clicking the location of the $X$ or responding target present without localizing the target. Observers were incentivized to identify the location of the $X$; they earned bonus points for a localization response. If observers successfully detected the target, they should have localized the $X$ to increase their gains. Even so, observers often failed to perform the localization response when responding target present. Additionally, these target-present without localization responses were more common in the high prevalence search task. Counter to the MDM's proposal, these data suggest that observers sometimes guessed the target was present after reaching their quitting thresholds and that this guessing strategy was informed by the prevalence rate of the target.

Here, we further investigate these target-present guessing strategies by manipulating both target prevalence and the number of items that an observer inspects before making a response. Through these manipulations, we can examine whether prevalence rate influences guessing and how information about the proportion of items in the display that have been ruled out as potential targets influences guessing rates. To illustrate, consider a block of trials with a $90 \%$ target prevalence rate. If the observer was required to make a response without inspecting any items, the observer should guess target present at least $90 \%$ of the time. However, if the observer had inspected almost all of the items in the display without finding a target and then was forced to make a response, the observer should be far less likely to guess target present. That is, the observer should adjust the base prevalence rates based on his or her accumulation of knowledge of the contents of the display.

\section{Method \\ Participants}

Forty undergraduates (30 female), ages 18 to 24 years, from Michigan State University's human subject pool participated for course credit. This sample size was chosen to match that of Schwark et al. (2013). All participants gave informed consent. All participants reported normal or corrected-to-normal vision.

\section{Stimuli}

The experiment was programmed using Experiment Builder from SR Research and used an EyeLink 1000 eye tracker. Stimuli were presented on a 21-inch monitor set to a resolution of $1024 \times 768$ and viewed from a chin rest at $55 \mathrm{~cm}$. Each trial consisted of an array of 24 items. Each item subtended $1.2^{\circ} \times$ $1.2^{\circ}$ of visual angle. The screen was divided into a $6 \times 4$ grid with an item appearing at a random location within each section of the grid (see Fig. 1). In target-absent trials, all 24 items were rotated offset $L$ s. In target-present trials, one randomly selected offset $L$ was replaced with a rotated $T$.

To create each search image, the screen area was divided into 24 (a $6 \times 4$ matrix) equal sized $\left(6.4^{\circ} \times 7.1^{\circ}\right)$ regions. A single item was placed within each region, with random jitter that allowed the item to appear anywhere within the region. This jitter broke up the orderly organization of the matrix and resulted in the items appearing in different locations across trials. The orientation of each item was randomly assigned.

\section{Procedure}

Participants were informed that on some trials the display would not terminate until they responded target present or absent, while on other trials, the display would automatically terminate after a short period. If the trial automatically terminated, they were to respond if the target was present or absent. After receiving these instructions, the eye tracker was calibrated to the subject using the nine-point calibration protocol of the EyeLink software. Next, the observer completed three blocks of trials in a randomized order. Each block had a different prevalence rate $(10 \%, 50 \%$, or $90 \%)$ and began with 50 practice trials to allow participants to adjust to the block's prevalence rate prior to data collection (Ishibashi, Kita, \& Wolfe, 2012; Wolfe \& Van Wert, 2010). There were 27 target-present trials per block.

Within a block, there were three trial termination conditions. A random one third were terminated by the participants'

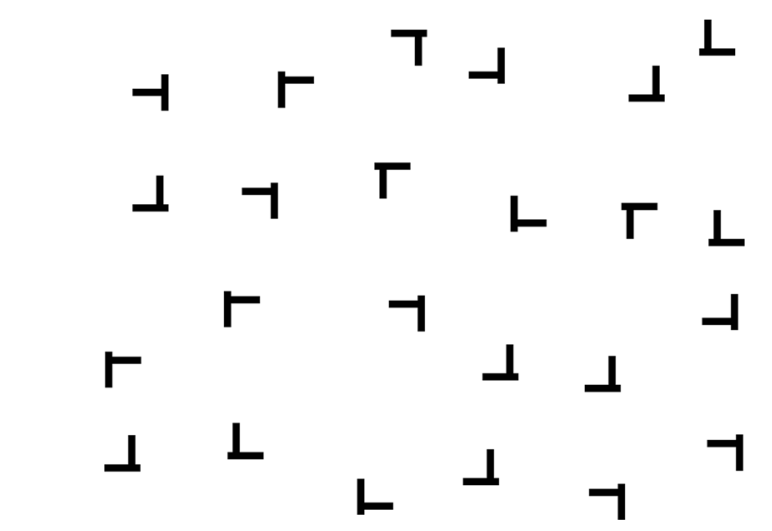

Fig. 1 Example array used during search task. Target $T$ is in the upperleft corner 
target-present/absent response. In the remaining trials, the search display disappeared after five or 10 fixations ( $1 / 3$ each), at which point the participant had to make their target-absent/ present judgement. There were an equal number of targetpresent trials in each termination condition with a prevalence block. All responses were followed by feedback.

\section{Results and discussion}

Data from one subject were removed from further analyses for the subject's failing to complete the experiment. Trials with a reaction time beyond the subject's mean plus 3 standard deviations for that prevalence rate were discarded from further analysis. Table 1 presents summary statistics for accuracy and reaction times for each prevalence block.

\section{Self-terminating trials}

An analysis of reaction time and accuracy for the selfterminating trials replicates standard prevalence effects. Only correct trials were included in the reaction time analysis. ${ }^{1}$ For reaction time (see Table 1), the main effects of target presence and prevalence and their interaction were all significant, all $F$ s $>15$, all $p \mathrm{~s}<.001$, showing that target-absent trials are faster at low prevalence. Similarly for accuracy, there was a significant Target Prevalence $\times$ Target-Present interaction, $F(2,76)$ $=25.25, p<.001, \eta_{\mathrm{p}}{ }^{2}=.399$, showing a higher miss rate at low prevalence, indicative of the prevalence effect. ${ }^{2}$

\section{Guessing}

To investigate observers' guessing rates, we analyzed falsealarm rates as a function of prevalence level and the amount of information from the array that was inspected prior to making a response. To precisely control the amount of information accrued in the five and 10 fixation conditions, we discarded trials where a false alarm was made before reaching the allowed number of fixations. ${ }^{3}$ This approach helped reduce the chance that a false alarm was due to misidentifying a distractor as a target, as an early termination by the observer

\footnotetext{
$\overline{1}$ Twelve observers do not have correct responses in either the target-absent high prevalence trials or the target-present low prevalence trials that were selfterminated. This is due to the limited number of trials contributing data to these cells. These observers are not included in the reaction time's repeatedmeasures analysis.

${ }^{2}$ Accuracy and false alarm data were arcsine-transformed (y' = $\arcsin (\operatorname{sqr}(\mathrm{y})))$ to account for unequal variances in binomial data (Hogg \& Craig, 1995). Mean and SEM data presented are the raw accuracy data.

${ }^{3}$ At low prevalence, $7 \%$ of 5 fixation trials were discarded and $21 \%$ of 10 fixation trials were discarded. At moderate prevalence, $8 \%$ of 5 fixation trials were discarded and $15 \%$ of 10 fixation trials were discarded. At high prevalence, $8 \%$ of 5 fixation trials were discarded and $18 \%$ of 10 fixation trials were discarded. The pattern of results is identical when these trials are included in the analyses.
}

Table 1 Means (and standard errors of the means) at each prevalence level in self-terminated trials

\begin{tabular}{llll}
\hline Prevalence & Presence & Accuracy & Reaction Time (ms) \\
\hline 10 & Present & $.443(.036)$ & $4083.49(151.00)$ \\
& Absent & $.986(.039)$ & $5398.75(208.68)$ \\
50 & Present & $.672(.033)$ & $4580.36(286.55)$ \\
& Absent & $.897(.035)$ & $6970.50(497.41)$ \\
90 & Present & $.823(.027)$ & $5538.88(270.02)$ \\
& Absent & $.718(.073)$ & $8702.54(654.81)$ \\
\hline
\end{tabular}

could indicate a false alarm based on an identification error rather than a target-present guess.

False-alarm data were submitted to a $3 \times 3$ repeatedmeasures ANOVA, with the factors target prevalence and fixations allowed. There were significant main effects of prevalence, $F(2,76)=40.61, p<.001, \eta_{\mathrm{p}}^{2}=.517$, and fixations allowed, $F(2,76)=38.12, p<.001, \eta_{\mathrm{p}}{ }^{2}=.501$. There was also a prevalence by fixations allowed interaction, $F(4,152)=6.67$, $p<.001, \eta_{\mathrm{p}}{ }^{2}=.149$, suggesting that the influence of prevalence rate is moderated by the amount of information accrued prior to making a guess. Specifically, the magnitude of the increase in false alarms caused by a high target prevalence rate was less pronounced for the unlimited fixations condition than the conditions that limited the number of fixations (see Table 2).

Three separate repeated-measures ANOVAs, one for each prevalence rate, confirm that false-alarm rates are influenced by the number of allowed fixations at all prevalence rates, all $F_{\mathrm{S}}>15.4$, all $p \mathrm{~s}<.001$, all $\eta_{\mathrm{p}}{ }^{2}>.29$ (see Fig. 2). Similarly, separate repeated-measures ANOVAs for each number of allowed fixations condition showed that false alarms increased as prevalence rate increased for all allowed fixations conditions, all $F \mathrm{~s}>4.9$, all $p \mathrm{~s}<.02$, all $\eta_{\mathrm{p}}{ }^{2}>.21$.

Overall, this pattern of results is consistent with people using a fairly sophisticated guessing strategy that is influenced by both the prevalence rate and the amount of information accrued prior to making a response. In addition, the interaction shows that this strategy weights the effect of prevalence rate by the amount of information accrued prior to making a guess.

\section{Ideal guessing}

To model ideal guessing, we used a basic computational model. First, we calculated the average number of uninspected items in each condition by subtracting the average number of items fixated per condition (see Table 3) from the total array size of $24 .{ }^{4}$

\footnotetext{
${ }^{4}$ It is important to note that allowing five or 10 fixations does not result in the observer inspecting five or 10 items. The observer could fixate on the same item more than once, or look at a space in the display, or the initial fixation following recalibration may not have fallen on an item. Also, we acknowledge that our model assumes that inspected distractors were correctly identified as distractors and that participants only had knowledge of fixated items.
} 
Table 2 Means (and standard errors of the means) of the probability of a false alarm at different levels of target prevalence and allowed number of fixations

\begin{tabular}{llll}
\hline Prevalence & 5 & 10 & Unlimited \\
\hline 10 & $.056(.008)$ & $.029(.006)$ & $.005(.003)$ \\
50 & $.34(.044)$ & $.22(.036)$ & $.06(.026)$ \\
90 & $.68(.076)$ & $.61(.079)$ & $.23(.068)$ \\
\hline
\end{tabular}

Next, we used prevalence rates to calculate the probability of each item in a display being a target. At $10 \%$ prevalence rate, each item had a $.4 \%$ chance of being a target (10\%/24 items). Similarly, each item had a $2.08 \%$ and $3.75 \%$ chance of being a target in the $50 \%$ and $90 \%$ target prevalence blocks, respectively. We then multiplied the probability of each item being a target by the number of uninspected items. We performed this calculation for each condition. The observers' guess rates and model predictions are presented in Fig. 2. As the figure shows, the general pattern of guesses is well characterized by this simple model. However, there is a systematic bias whereby participants are always less likely to guess that the target is present that the model predicts.

Why might this systematic bias appear in our data? One possible explanation is that people are confident that they would have found the target fairly early in their search. According to influential models of visual search (Chun \& Wolfe, 1996; Wolfe, 1994), an initial parallel stage of search assigns each item an activation level based on the items similarity to the target. The subsequent sequential search process investigates individual items in order of priority based on this activation level. Items most likely to be the target should be inspected early in the search process. If observers factored this into their guessing rate, they should be somewhat confident that the first few items inspected were those that were mostly likely to be the target. If these initial items were not the target,

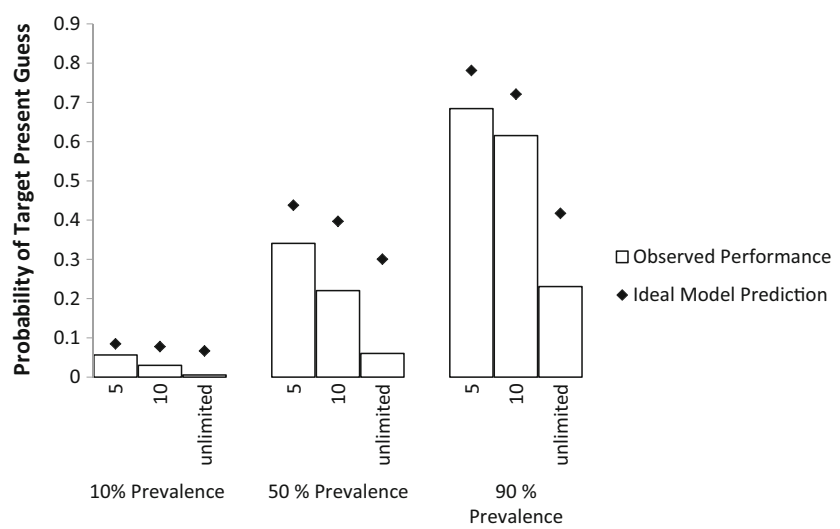

Fig. 2 Given the target prevalence rate and allowed number of fixations, the probability of a target-present guess. White bar represents observers' actual guessing rates. Black diamond represents probability of a target's presence given the target prevalence rate and the number of items inspected
Table 3 Means (and standard errors of the means) of the number of unique items inspected at each level of prevalence and number of allowed fixations

\begin{tabular}{llll}
\hline Prevalence & 5 & 10 & Unlimited \\
\hline 10 & $2.77(.126)$ & $4.55(.22)$ & $7.26(.51)$ \\
50 & $2.94(.18)$ & $4.91(.30)$ & $9.55(.93)$ \\
90 & $3.16(.36)$ & $4.77(.45)$ & $12.87(1.02)$ \\
\hline
\end{tabular}

Note. The number of unique items inspected at five and 10 allowed fixations did not significantly differ between prevalence rates (all $p \mathrm{~s}>$ .14). Though the number of unique items inspected during unlimited fixations differed (all $p \mathrm{~s}<.002$ ), this was expected as a result of target prevalence causing a shift in quitting threshold

the observer should be somewhat more confident that the target is not present than our simple model, which assigns each item an equal probability of being the target, suggests. This process would produce the systematic bias we observe.

\section{Discussion}

We found standard prevalence effects in the reaction time and accuracy data; as prevalence decreased, target-absent reaction time decreased and miss rates increased.

More important for our current question were the falsealarm data. We analyzed false alarms as a function of target prevalence; high, moderate, or low. The data show as prevalence increases, so too do false alarms. Our analysis replicates Schwark et al. (2013) in that we both find evidence suggesting increasing target prevalence increases target-present guessing. Our findings are inconsistent with the claims of the MDM that a target-absent response will always be made if the target is not identified prior to making a response. Instead, our finding that prevalence rate directly impacts guessing behavior is consistent with the claims of Schwark et al. (2013), although we use a different method to reach the same conclusion.

We sought to expand on the results of Schwark et al. (2013) in two ways; first, by using stimuli that were easier to identify, and second by controlling for the amount of information an observer accumulated before terminating search. By using relatively simple $T \mathrm{~s}$ and $L \mathrm{~s}$ as search stimuli, we reduced the probability of misidentifications. Additionally, our stimuli did not overlap, again reducing the possibility of misidentifications, which makes the false alarms more attributable to target-present guessing than identification errors, allowing a more direct test of our theory. These stimuli contrast with realworld stimuli where stimuli may be less clear, and the observer may not know exactly what they are looking at. Under those real-world circumstances of uncertainty, prevalence rate may have a stronger effect on decision making, and accumulated information would be less important when the quality of information is low. Future research should address these 
concerns by manipulating accumulated information in a more difficult search task.

Trials ended automatically did not allow us to test how observers would respond when a trial is self-terminated, but it did allow us to experimentally test the contribution of accumulated information to guess rate. By automatically terminating search based on number of fixations, we show that the amount of information the observer accumulates prior to making a response moderates the effect of prevalence on guessing. When an observer is allowed to inspect many items in the display, the prevalence rate becomes less impactful. Instead, the observer appears to weight the prevalence rate by the amount of accumulated information. Indeed, the observed guessing rates were very similar to the ideal guessing rates based on the probability of a target being present, but uninspected, before the trial terminates. However, observers were slightly more likely to respond target absent than a model based on strict probabilities would predict. One explanation for this bias is that observers may have been confident in their initial guidance to a target when present. If so, the weights due to inspecting items would not be strictly linear, with higher weighting on earlier fixations. This type of weighting could produce the bias toward target-absent responses.

Although our use of automatically terminated trials shows that this has a strong effect of accumulated evidence on guessing rate, there is the possibility that the process that occurs during automatically terminated trials is different from that during self-terminated trials. That is, by automatically terminating trials, we may have forced people into an atypical guessing mode that does not occur when one self-terminates a trial, and the influence of accumulated information may only appear in this atypical situation.

One possible way to evaluate this is to determine whether there is evidence that the amount of accumulated information impacts the likelihood of target-present guess in selfterminated trails. Some data bear on this question. We have data from the unlimited condition, and we have data from a subset of trials in the automatically terminated conditions in which participants self-terminated the trial prior to reaching the automatic cutoff. Since prevalence rate can impact target guessing rates (Shwark et al., 2013), and can impact the likelihood of misidentifying a distractor as a target (Godwin, Menneer, Riggs, Cave, \& Donnelly, 2014; Peltier \& Becker, 2016), to evaluate the impact of accumulated information, we collapsed across prevalence rates. On average, the amount of information accumulated (as indexed by number of fixations) prior to making these self-terminated responses differed by allowed fixations condition, $F(2,34)=64.23, p<.001, \eta_{\mathrm{p}}{ }^{2}=$ .79. For these self-terminated trials, there were more fixations in the unlimited condition $(M=14.02, S E M=1.23)$ than the 10 (30 observers made a premature response when ten fixations were allowed) $(M=5.51, S E M=.47)$ fixation cutoff condition, $t(29)=9.6, p<.001 d=3.55$, and there were more in the 10 than the five (18 observers made a premature response when five fixations were allowed) $(M=2.48, S E M=$ .26) fixation cutoff condition, $t(17)=10.23, p<.001, d=4.96$. Thus, for these trials, if accumulated information influenced guessing, we would predict fewer target-present guesses in the unlimited than the 10 fixation cutoff condition, with fewer still in the five fixation cutoff condition. By contrast, if the explanation for target-present guesses was that people misidentified a distractor as a target, we would expect the opposite pattern, more target-present guesses in the unlimited than the 10 fixation cutoff condition, and the fewest in the five fixation cutoff condition, since the opportunity to make such an error decreases as the number of inspected items decreases. To evaluate this issue, we collapsed across subjects and prevalence rates and looked at the percentage of self-terminated trials that were target-present guesses (false alarms), as a function of the allowed fixations condition. In doing so, we find that participants made a target-present guess on only $1.3 \%$ (46 false alarms out of 3,539 self-terminated unlimited fixation trials) of trials in the unlimited case, $4.35 \%$ (31 false alarms out of 713 self-terminated 10 fixation trials) of the self-terminated trials in the 10 fixation condition, and $7.45 \%$ (19 false alarms out of 255 self-terminated five fixation trials) of selfterminated trials in the five fixation cutoff condition. A chi square test comparing these rates was significant, $\chi^{2}(2)=$ $48.89, p<.001$. This pattern is completely consistent with our claim that accumulated information impacts guess rate, even in these self-terminated trials, and is the opposite pattern that one would expect if guesses were simply a result of misidentifying a distractor as a target.

In sum, it appears that people do make target-present guesses, and that the probability of doing so is impacted both by the base probability of the target being present (the prevalence rate) and the amount of information accumulated prior to making a response. The more information that is accumulated, the less influence the prevalence rate has. This suggests that observers adopt a somewhat sophisticated guess strategy.

\section{References}

Chun, M., \& Wolfe, J. M. (1996). Just say no: How are visual searches terminated when there is no target present? Cognitive Psychology, 30. doi:10.1006/cogp.1996.0002

Godwin, H. J., Menneer, T., Riggs, C. A., Cave, K. R., \& Donnelly, N. (2014). Perceptual failures in the selection and identification of lowprevalence targets in relative prevalence visual search. Attention, Perception, \& Psychophysics. doi:10.3758/s13414-014-0762-8

Hogg, R. V., \& Craig, A. T. (1995). Mathematical statistics. Englewood Cliffs, NJ: Prentice-Hall.

Hout, M. C., Walenchok, S. C., Goldinger, S. D., \& Wolfe, J. M. (2015). Failures of perception in the low-prevalence effect: Evidence from active and passive visual search. Journal of Experimental 
Psychology: Human Percepteption \& Performance, 41(4), 977994. doi:10.1037/xhp0000053

Ishibashi, K., Kita, S., \& Wolfe, J. M. (2012). The effects of local prevalence and explicit expectations on search termination times. Attenion, Perception, \& Psychophysics, 74(1), 115-123. doi:10. 3758/s13414-011-0225-4

Peltier, C., \& Becker, M. W. (2016). Decision processes in visual search as a function of target prevalence. Journal of Experimental Psychology: Human Percepteption \& Performance. doi:10.1037/ xhp0000248

Rich, A. N., Kunar, M. A., Van Wert, M. J., Hidalgo-Sotelo, B., Horowitz, T. S., \& Wolfe, J. M. (2008). Why do we miss rare targets? Exploring the boundaries of the low prevalence effect. Journal of Vision, 8(15), 15.
Schwark, J., Macdonald, J., Sandry, J., \& Dolgov, I. (2013). Prevalencebased decisions undermine visual search. Visual Cognition, 21(5). doi:10.1080/13506285.2013.811135

Van Wert, M. J., Horowitz, T. S., \& Wolfe, J. M. (2009). Even in correctable search, some types of rare targets are frequently missed. Attention, Perception, \& Psychophysics, 71(3), 541-553. doi:10. 3758/APP.71.3.541

Wolfe, J. M. (1994). Guided search 2.0 a revised model of visual search. Psychonomic Bulletin \& Review, 1(2), 202-238.

Wolfe, J. M., Horowitz, T. S., \& Kenner, N. M. (2005). Rare items often missed in visual searches. Nature, 435(26), 439-440.

Wolfe, J. M., \& Van Wert, M. J. (2010). Varying target prevalence reveals two dissociable decision criteria in visual search. Current Biology, 20(2), 121-124. doi:10.1016/j.cub.2009.11.066 